\title{
Assessing the Kinect's Capabilities to Perform a Time- based Clinical Test for Fall Risk Assessment in Older People
}

\author{
Jaime A. Garcia ${ }^{1}$, Yusuf Pisan ${ }^{2}$, Chek Tien Tan ${ }^{2}$, Karla Felix Navarro ${ }^{1}$ \\ ${ }^{1}$ mHealth Lab - iNEXT Reasearch Group, ${ }^{2}$ Games Studio, \\ University of Technology Sydney, \\ Australia \\ Jaime.A.GarciaMarin@student.uts.edu.au, \{Yusuf.Pisan, \\ ChekTien.Tan, Karla.FelixNavarro\}@uts.edu.au
}

\begin{abstract}
The Choice Stepping Reaction Time (CSRT) task is time-based clinical test that has shown to reliably predict falls in older adults. Its current mode of delivery involves the use of a custom-made dance mat device. This mat is a measurement tool that can reliably obtain step data to discriminate between fallers and non-fallers. One of the pitfalls of this test is that the technology in use still imposes an obstacle on the degree of freedom to be able to perform adaptive exercises suitable for the elderly. In this paper, we describe a Kinectbased system that measures stepping performance through the use of a hybrid version of the CSRT task. This study focuses on assessing this system's capabilities to reliably measure a time-based clinical test of fall risk. Results showed a favorable correspondence and agreement between the two systems, suggesting that this platform could be potentially useful in the clinical practice.
\end{abstract}

Keywords: Kinect, Elderly, Fall Risk Assessment, Reaction Time Test, Stepping Performance.

\section{Introduction}

Since the release of the Microsoft Kinect, both research communities and industry have been actively investigating its potential use in the area of aged care and rehabilitation. This is mainly due to its capability to track real-time full body movements in 3D, a characteristic that was not available in early consumer game technologies such as the Nintendo Wii or the PS3. In the area of fall prevention and safety for the elderly, the Kinect has also gained much interest. Kinect-based applications range from health and home monitoring systems [13], through unobtrusive fall detection platforms [9]; coaching, rehabilitation and therapeutic tools[1], fall prevention training systems [7], to fall risk assessment tools [16]. Kinect-based serious games, being one of the most popular approaches, have shown a positive acceptance among seniors [6]. The fun factor inherent in such games and their ability to promote physical move- 
ments are ideal to encourage the elderly to exercise [4]. An example can be seen in the work done by Kim et al. [10], where the use of a commercial Kinect game showed to improve hip muscle strength and balance control in older adults after completing an 8 -weeks intervention.

From a clinical perspective, the capabilities of the Kinect have also the potential to implement low-cost methods to assess fall risk in older adults. This is achieved through the collection of measurement that fulfill the requirements of certain clinical tests such as posture control [2], gait [15], dual tasking ability [8], and mobility [11], among others. Overall, these methods rely on the Kinect to obtain information of the human body positions in real-time, which are shown to be fairly accurate. For instance in the work done by Dutta et al [3], the Kinect demonstrated the ability to validly assess kinematic strategies of postural control. This suggests that the Kinect could be considered an effective tool when it comes to collection position-based measurements. However, for time-based measurements the Kinect introduces an additional challenge as it is a camera-based device restricted to process up to 30 skeleton frames per second.

The work presented in this paper focuses on evaluating the capabilities of the Kinect to reliably collect timing variables to fulfill the requirements of the Choice Stepping Reaction Time (CSRT) task, a time-based clinical test that has shown to reliably predict falls in order adults [12]. This study hence evaluates the validity of a Kinectbased system developed by the authors with a validated choice reaction time device. The latter has shown to effectively discriminate between groups of recurrent fallers and non-fallers [14]. The rest of the paper is structured as follows. Section 2 presents brief summary of related work in the field of fall risk assessment and describes the Choice Stepping Reaction time. Section 3 sets out the methodology used for this evaluation. Results and Discussion can be found in Section 4 and 5. Finally, conclusions and future work are presented in Section 5.

\section{Related Work}

The Choice Stepping Reaction Time (CSRT) task is a composite measure of sensorimotor functions, such as balance and strength, and cognitive functions such as attention and central processing speed [12]. The test is able to combine several dimensions of fall risk based on these composite metrics. For the CSRT Task, the person stands on the two central step panels of a wooden board (see Figure 1). One of four surrounding panels (front left or $L F$, front right or $R F$, left or $L L$, right or $R R$ ) illuminates randomly and the person is required to step on this panel as quickly as possible and then return to the center. The sequence is presented randomly as well as the time between trials so that the user is unable to anticipate the time and location of the next stimulus. The mean reaction time of 20 trials is then measured and analyzed for clinical diagnosis.

In the work done by Schone et al. [14], a Dance Mat Choice Step Reaction Time device was introduced and validated against the original CSRT Test. In this system, the CSRT Test is achieved through the use of a custom-made dance mat (or MAT) 
that contains 12 step panels. The mechanics of the test are the same as the original version of the CSRT, however, visual stimuli are presented on the LCD monitor and the user is expected to step accordingly on respective positions on the MAT (see Figure 4). In this work, the MAT showed high correlations with the laboratory-based measure of the CSRT test. Also, the ability to reliably differentiate between fallers and non-fallers was also confirmed. However, one of the pitfalls of this work is that the use of the Dance Mat still imposes an obstacle on the degree of freedom and does not allow for the collection of spatial parameters.

In [5], the authors introduced StepKinnection ${ }^{\mathrm{TM}}$, a system that uses the Microsoft Kinect's depth and motion capture technology to measure stepping performance through the use of a hybrid version of the Choice Stepping Reaction Time (CSRT) task. In this system, the person stands in front of a computer screen or TV connected to a Kinect PC. The representation of the player in the system is pair of shoes mirroring the person's feet movements (see Figure 2). Six symmetrically distributed squareshaped virtual panels are drawn on the screen representing the step panels surrounding the person. The mechanics of the test are the same as the original version of the CSRT with the exception that the person steps in space (see Figure 3).

User's actions such as a 'step' or a 'foot liftoff' are recognized by translating the user's lower limb movements obtained by the Kinect's depth sensor into the gamelike platform. The system continuously retrieves spatial data (or skeleton frames) to determine whether a foot is intersecting one of the virtual panels. When the intersection of a foot and one panel is detected, the system records this action along with a timestamp. These actions are subsequently used for the calculation of the following time-based variables which are essential in the completion of the CSRT test: (1) Decision Time (DT): time elapsed between the instance where the sector turns green and the player lifts his/her foot off the central panel, (2) Movement Time (MT): time it takes for the user to step on a coloured sector once leg movement is initiated; (3) Response time (RT): Decision Time (DT) + Movement Time (MT). In addition to this, complementary spatial parameters are also captured for a more descriptive assessment of fall risk. These are: (1) Active Foot Positioning (AFP): (x,y,z) coordinates of the user's foot when stepping on a panel; (2) Observed Step Length (SL): distance between left and right foot while stepping on a panel; (3) Stepping Accuracy Coefficient (SAC): Difference between expected step length and observed step length.

The ability to collect these measures simultaneously makes this system potentially useful in a clinical setting as it can evaluate several dimensions involved in the assessment of fall risk in older people. However, since the Kinect is a camera-based device restricted to process 30 skeleton frames per second, the accuracy of the mentioned time-based measurements needs further evaluation. 


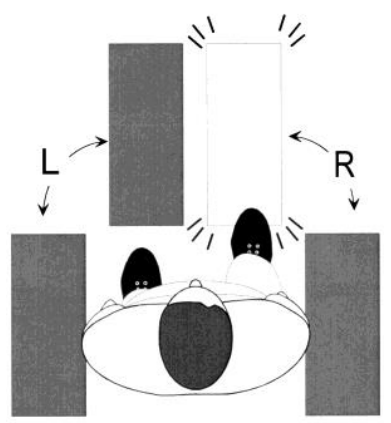

Fig. 1. Original version of the Choice Stepping Reaction Time (CSRT) test [12].

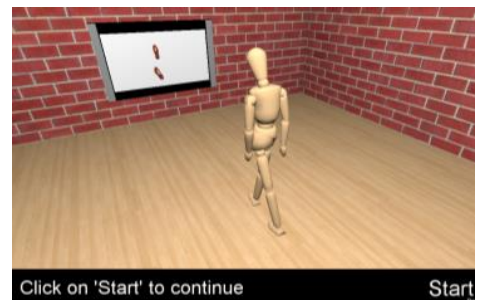

Fig. 2. Interaction with the Kinect-based CSRT test [5].

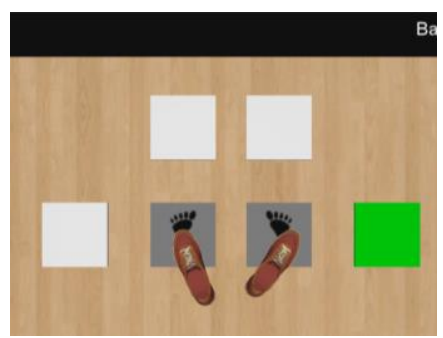

Fig. 3. Mechanics of Kinect-based CSRT Test [5].

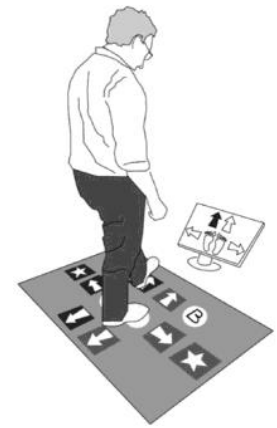

Fig. 4. MAT version of the CSRT Test [14]

Furthermore, the work presented in this paper focuses on the assessment of Kinect's capabilities for perform the CSRT test. This study aims to find out whether the limitation imposed by the frame rate is determinant when collecting time-based measurements associated to human movements. The following section sets out the methodology used for this evaluation.

\section{$3 \quad$ Methodology}

A convenient sample of 10 individuals was recruited. Participants consisted of research students and lecturers from the University Technology Sydney. The eligibility criteria are described as follows: (1) Not contributed to the design of these instruments. (2) Able to walk independently without assistance, and (3) Fluent in the English language.

Participants were asked to perform the CSRT test on both systems. The order was randomized to counterbalance order effects. Prior to testing on the Kinect, each participant went through a calibration process in order to determine the proper floor plane $^{1}$ and the participant's height ${ }^{2}$. For both systems, participants were given a prac-

1 To minimize bias in data collection due uneven surfaces or placing the Kinect on a slightly tilted table, a short calibration sequence was incorporated to determine the orientation of the actual floor plane (or ground). 
tice trial in order to familiarize with the mechanics of the test and to confirm the fully understanding of the instructions. Later, they were instructed to complete a full CSRT task where execution of the test was recorded by the corresponding controlling software. Once the test was completed, they were asked to complete a practice trial and a full CSRT task on the other platform. In this experiment, the software that controls the MAT and the Kinect were run in the same computer to minimize bias in the measurements due to variability in computational power. As instructed in the original version of the CSRT test, the mean Reaction Time (RT) of 20 trials is measured and analyzed. Also, the mean Decision Time (DT) and Movement Time (MT) were examined for a more descriptive assessment.

In order to determine the validity of the Kinect-based CSRT, this evaluation was conducted in two steps. Firstly, the consistency and association of the three parameters (DT, MT and RT) per participant per panel was assessed. Shapiro-Wilk test was utilized to assess the distribution of the time-based variables. The consistency and association of the three parameters (DT, MT and RT) were assessed by calculating the Pearson Correlation Coefficient and the Intraclass Correlation Coefficient model 3,1. Secondly, the RT values were used to compute the CSRT test for both the Kinect and MAT and a similar analysis was then conducted.

\section{Results}

Regarding to the consistency of the Reaction Times (RTs), the Shapiro-Wilk test showed normality across the log transformed time-based measures of the CSRT (Sig. $>$ 0.05). The Pearson Correlation Coefficient showed high association between the values collected by Kinect and the MAT (Pearson $r=.746$ ) (Refer to Table 1). Likewise, the ICC model 3,1 confirmed the consistency and agreement of the RT values across the samples (ICC Single Measures $=.657$, Average Measures $=.793$, Sig = $.000)$.

Regarding the consistency of the Decision Times (DT) and Movement Times (MT), complementary parameters computed on the MAT version of the CSRT test; the Pearson $r$ analysis determined that the DT values were highly correlated; however, the MT values reported a poor association. (Refer to Table 1). Finally, the Choice Stepping Reaction Time (CSRT) test was calculated for both the Kinect and MAT as instructed in its original version. The Pearson $r$ analysis showed high association between the CSRT values obtained by the Kinect and the MAT (Refer to Table 2). Similarly, the ICC model 3,1 confirmed the consistency and agreement between the CSRT measures computed with the Kinect and the MAT. (ICC Single Measures $=.645$, Average Measures $=.784$, Sig .016).

2 The participants' height is also measures and used to determine the distance of the panels (in this paper known as Expected Step Length). This makes the test equally challenging for audiences as panels are dynamically located based on the user's height which also takes away the limitation of the original test where the step panels are fixed. 
Table 1. Correlation of Timing Variables (per Panel).

\begin{tabular}{llll}
\hline System & Panel & Outcome & Pearson r \\
\hline Kinect-Mat & LF & DT & .6110 \\
& MT & .2100 \\
& RT & $\mathbf{. 7 3 2 0}$ \\
\cline { 2 - 4 } & LL & DT & .4350 \\
& MT & .1230 \\
& RT &. $\mathbf{7 5 5 0}$ \\
\cline { 2 - 3 } & RF & DT & .7930 \\
& MT & .7060 \\
& RT & .7980 \\
\cline { 2 - 3 } & RR & DT & .7030 \\
& MT & .1570 \\
& RT & $\mathbf{. 7 6 8 0}$ \\
\hline
\end{tabular}

Table 2. Correlation of CSRT, MT and DT

\begin{tabular}{lll}
\hline System & Outcome & Pearson r \\
\hline Kinect-Mat & CSRT & $\mathbf{. 7 8 7 5}$ \\
& MT & .3329 \\
& DT & .6430 \\
\hline
\end{tabular}

\section{Discussion}

This study shows that our Kinect-based system is able to compute the Choice Stepping Reaction Time (CSRT) task. Timing variables such as Decision Time (DT), Movement Time (MT) and Reaction Time (RT) were analised to assess the capabilities of the Kinect to reliably perform a time-based clinical test. The variability of the reaction times was assessed and reported to be equivalently distributed in the Kinect and the MAT. Correlations between the Kinect and the MAT were high for RT suggesting that the Kinect could fulfill the requirements of the CSRT Test. In regards to the DT and MT values, the former showed high correspondence between the two systems whereas the latter reported a poor correlation. It is likely that the MT value did not agree completely due to the lack of precision in detecting the initiation of the user's leg movement. In the Mat this is determined by absence of pressure on the step panels whereas in the Kinect is determined by the location of the foot in relation to the virtual panel, making it difficult to detect such a fine movement. In spite of this, the RT values were utilized to compute measure of the CSRT task. Statistical analysis and correlation demonstrated that Kinect measure of the CSRT confidently agreed with the CSRT values computed by the MAT. In addition to this, the data presented show that RT for the Kinect was overall shorter than for the MAT as shown in Figure 5 , suggesting that this test might be slightly less cognitively demanding. It is likely that the immersive nature of this system and the appropriate provision of real-time feedback remove the extra processing time required to operate with the MAT (where 
stimuli are presented on the screen and the execution is expected to happen on the dance mat). Alternatively, participants may have felt more confident to step faster in space due to the absence of wearable sensors or a physical apparatus.

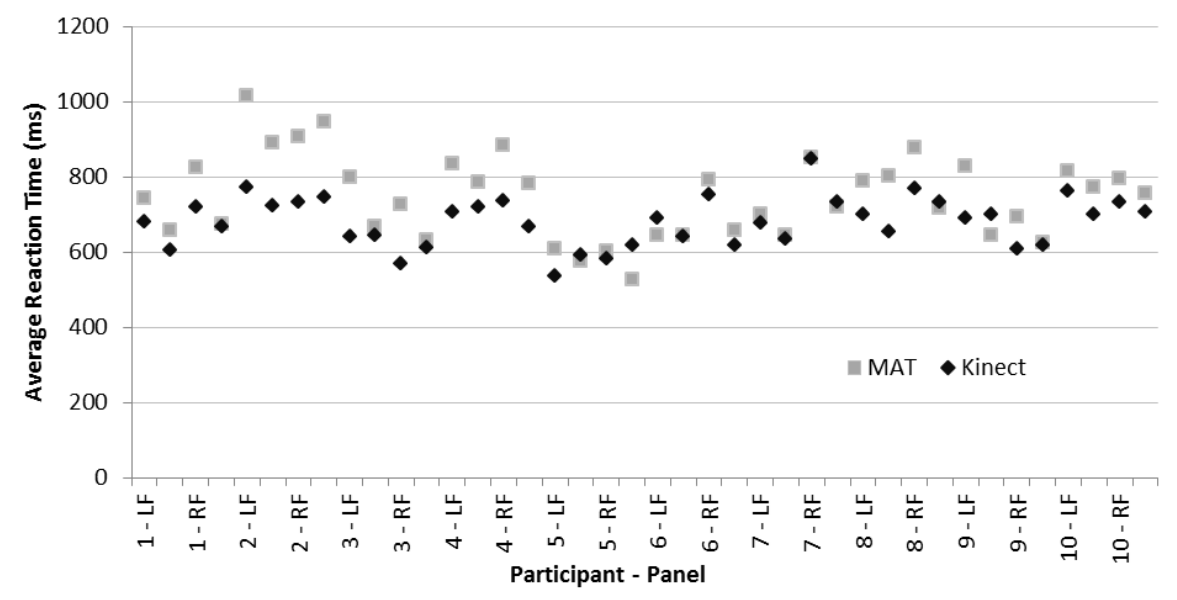

Fig. 5. Comparison between Average Reaction Times obtained with the Kinect and the MAT per Participant per Panel.

\section{Conclusion and Future Work}

This paper describes an evaluation where we assessed the ability of a Kinect-based system to reliably perform the Choice Stepping Reaction Time (CSRT) task. This study involves a technical assessment where the Kinect time-based measurements were compared with a validated reaction time device that has proved to reliably discriminate between fallers and non-fallers. The results showed high association between the Reaction Times (RT) and Decision Times (DT) values obtained in both systems. Movement Time (MT) however did not show agreement most likely for the limitations in determining the initiation of the user's leg movement. Finally, RT values were utilized to compute the CSRT test in order to assess the responsiveness of the system. Statistical analysis showed that both systems correlate favorably, suggesting that the Kinect system might be valid and reliable. More importantly, this evaluation suggests that this Kinect-based system has the potential to be used in a clinical setting as it can consistently measure both spatial and time based parameters required for the fulfillment of a validated clinical test. Yet, the system's capability to reliably discriminate between fallers and non-fallers still requires further evaluation. The next stage of this study will involve the clinical validation of the step parameters, followed by the conduction of controlled trials to determine the ability of the system to discriminate between groups of fallers and non-fallers. 


\section{References}

1. Chen S-T, Huang Y-G, Chiang I-T: Using somatosensory video games to promote quality of life for the elderly with disabilities. In: 2012 IEEE Fourth International Conference on Digital Game and Intelligent Toy Enhanced Learning (DIGITEL), p 258-262 (2012)

2. Dutta A, Banerjee A, Dutta A: Low-cost visual postural feedback with Wii Balance Board and Microsoft Kinect-a feasibility study. In: Point-of-Care Healthcare Technologies (PHT), p 291-294 (2013)

3. Dutta T: Evaluation of the Kinect ${ }^{\mathrm{TM}}$ sensor for 3-D kinematic measurement in the workplace. Applied ergonomics 43:645-649 (2012)

4. Ganesan S, Anthony L: Using the kinect to encourage older adults to exercise: a prototype. In: Proceedings of the 2012 ACM annual conference extended abstracts on Human Factors in Computing Systems Extended Abstracts. ACM, p 2297-2302 (2012)

5. Garcia JA, Pisan Y, Tan CT et al.: Step kinnection: a hybrid clinical test for fall risk assessment in older adults. In: CHI '14 Extended Abstracts on Human Factors in Computing Systems. ACM, Toronto, Ontario, Canada, p 471-474 (2014)

6. Gerling K, Livingston I, Nacke L et al.: Full-body motion-based game interaction for older adults. In: Proceedings of the 2012 ACM annual conference on Human Factors in Computing Systems. ACM, p 1873-1882 (2012)

7. John M, Klose S, Kock G et al.: Smartsenior's interactive trainer-development of an interactive system for a home-based fall-prevention training for elderly people. In: Ambient Assisted Living. Springer, p 305-316 (2012)

8. Kayama H, Okamoto K, Nishiguchi $\mathrm{S}$ et al.: Concept Software Based on Kinect for Assessing Dual-Task Ability of Elderly People. GAMES FOR HEALTH: Research, Development, and Clinical Applications 1:348-352 (2012)

9. Kepski M, Kwolek B: Fall detection on embedded platform using kinect and wireless accelerometer. In: Computers Helping People with Special Needs, p 407-414 (2012)

10. Kim J, Son J, Ko N et al.: Unsupervised Virtual Reality-Based Exercise Program Improves Hip Muscles Strength and Balance Control in the Elderly: A Pilot Study. Archives of physical medicine and rehabilitation(2012)

11. Lohmann O, Luhmann T, Hein A: Skeleton Timed Up and Go. In: 2012 IEEE International Conference on Bioinformatics and Biomedicine (BIBM), p 1-5 (2012)

12. Lord SR, Fitzpatrick RC: Choice Stepping Reaction Time: A Composite Measure of Falls Risk in Older People. Journals of Gerontology Series A: Biological Sciences \& Medical Sciences 56A:M627 (2001)

13. Parajuli M, Dat T, Wanli M et al.: Senior health monitoring using Kinect. In: 2012 Fourth International Conference on Communications and Electronics (ICCE), p 309-312 (2012)

14. Schoene D, Lord SR, Verhoef $P$ et al.: A novel video game-based device for measuring stepping performance and fall risk in older people. Archives of physical medicine and rehabilitation 92:947-953 (2011)

15. Stone E, Skubic M: Evaluation of an inexpensive depth camera for in-home gait assessment. Journal of Ambient Intelligence and Smart Environments 3:349-361 (2011)

16. Stone EE, Skubic M: Evaluation of an inexpensive depth camera for passive in-home fall risk assessment. In: 2011 5th International Conference on Pervasive Computing Technologies for Healthcare (PervasiveHealth), p 71-77 (2011) 